تفعيل وظيفة المراجعة الااخلية في ظل بيئة نظم تخطيط موارد المنظمة (دراسة ميدانية )

خالا مصباح مصطفى

باحث ماجستير
د/ احمد علي غازي

أستاذ المحاسبة المساعد غأي

كلية التجارة - جامعة المنصورة

\section{ملذص البحث:}

نتيجة التطور الهائل في تكنولوجيا المعلومات ودورها الفاعل في إنجاز المهام بشكل أفضل في كافة أنشطة المنظمة وظهور ما يسمى بنظم تخطيط موارد المنظمة Enterprise Resource Planning الحاجة إلي تطوير وظيفة المراجعة الداخلية والتي تم انتقادها لسنوات طويلة

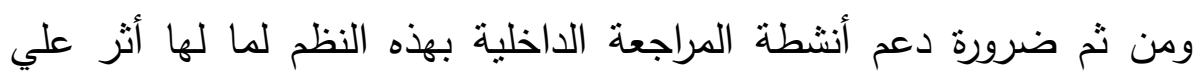
تحسين وظيفة المراجعة الداخلية. ومن هنا تمثل الهدف البحث في تفعيل وظيفة المراجعة الداخلية في ظل بيئة نظم تخطيط موارد المنظمة.

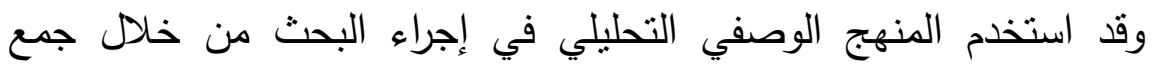

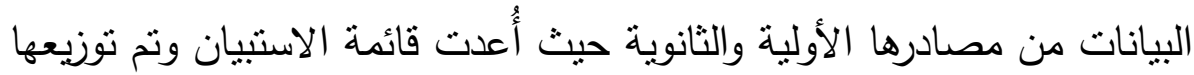
على مجتمع البحث المتمنل في بعض من موظفي المصارف العاملة في ليبيا

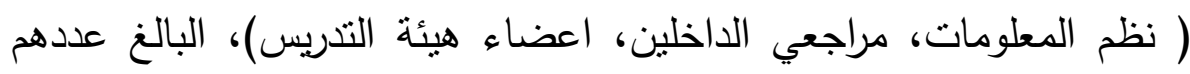
ونم استخدام برنامج التحميل الاحصائي (VA) الدارسة، وتم التوصل إلي النتائج الرئيسية النالية :

* وجود اتفاق معنوي بين فئات البحث حول تفعيل وظيفة المراجعة الداخلية في ظل بيئة نظم تخطيط الموارد المنظمة، وبدوره يؤدي لدعم استقالالية

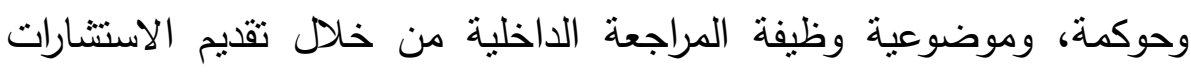
والتأكيدات للإدارة وخاصة فيما يتعمق بالتخصيص الأمثل للموارد المتاحة. 
* وجود اتفاق معنوي بين فئات البحث حول مدى ضرورة نوفر مجموعة من

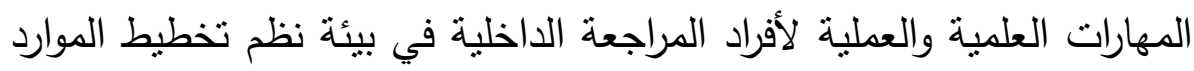

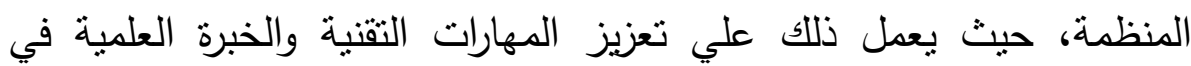

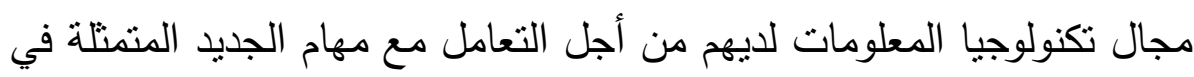
نظم تخطيط الموارد.

$$
\text { واوصى البحث علي التالي: }
$$

* ضرورة الاتجاه نحو تطبيق منهجية المراجعة المستمرة ( مدخل المراجعة

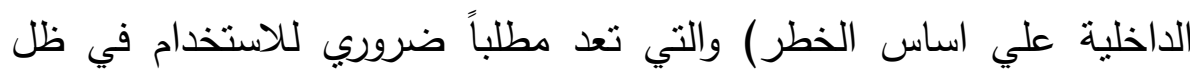
تطبيق نظم تخطيط المواردERP ، حيث تمكن المنظمة من تخفيف المخاطر التي تصاحب تطور تكنولوجيا المعلومات، وأن تتوفر الرغبة لدى المنظمات في تحمل تكاليف تدريب العامين، والدعم الكامل من الإدارة والمساهمين لمنهجية

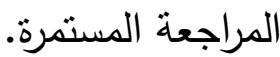

* ضرورة تتمية مهارات المراجعين الداخليين من خلال الدورات التدريبية

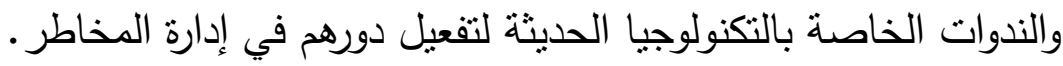
الكلمات الاساسية: المراجعة الداخلية علي اساس الخطر - نظم تخطيط الموارد - ERP

\section{Abstract:}

As a result of the tremendous development in information technology and its role in the effective fulfillment of the tasks better in all the activities of the Organization, and the emergence of the so - called planning systems of the resources of the Organization (ERP) Enterprise Resource Planning, which increased the need for the development of the internal audit function, which was criticized for long years, and thus the need to support 
the activities of the internal audit of these systems to have an impact on the improvement of the internal audit function And here the aim is the search in the activation of the internal audit function in an environment planning systems of the resources of the Organization.

And may use descriptive approach the analytical work in the research through the collection of data from primary sources and secondary school has prepared a list of the questionnaire was distributed to the community of some of the staff of the banks operating in Libya (information systems, board entering, members of the teaching staff) numbering (87) and the use of the statistical analysis SPSS.

Search reached the following conclusion:

* The existence of moral agreement between the categories of research on the activation of the internal audit function in an environment of resource planning systems of the Organization, in turn, lead to support the independence and corporate governance and objectivity of the internal audit function through the provision of consultations and assurances of will, especially with regard to the optimum allocation of available resources.

* The existence of moral agreement between the categories of research on the extent of the need to provide a range of skills, both scientific and practical members of internal audit in a resource planning systems organization, where they serve to enhance the technical skills and practical experience in the field of information technology have to deal with the new tasks of resource planning systems.

Research recommended the following:

* The necessity of the trend toward the application of the methodology for the continuing review (the entrance of Risk based internal auditing) which is an essential requirement 
for use under the application of the Enterprise Resource Planning ERP, where the organization has been able to reduce the risks associated with the development of the information technology and the desire to defray the costs of the training of personnel, and the full support of the administration and shareholders of the methodology of ongoing review.

* The need to develop the skills of internal auditors through training courses and symposia on modern technology to activate their role in the management of risks.

Keywords: Risk based internal auditing - Enterprise Resource Planning (ERP) - Risk management.

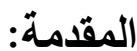

أظهرت أحدث التغييرات في بيئة الأعمال احتياج المنظمات لطرق جديدة

لتحسين وتطوير مهام المنظمات، حيث توفر تكنولوجيا المعلومات الأدوات اللازمــة للمنظمـات للاسـتجابة بفعاليــة وكفــاءة، مـن ناحيـة أخـرى لا تـزال

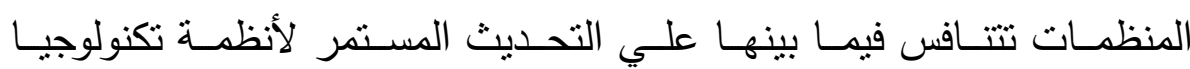
المعلومـات الجديدة من أجل الحفاظ على الميزة التتافسية في بيئة الأعمال المتتامية.

ونتيجة للانتتار الواسع لتكنولوجيا المعلومات والتي تعتبر مطلبا أساسياً من متطلبات بيئة الأعمال المعاصرة، ونظرا لدورها في إنجاز المهام بشكل أفضل

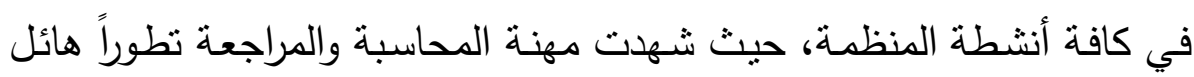
في ذلك المجال والذي ترتب عليه ظهور مـا يسمى بنظم تخطيط الموارد وهي نظم معلومات المنظمـة ERP Enterprise Resource Planning متكاملـة علي مستوى المنظمـة تربط إدارتها وأقتسامها بقاعدة بيانات مركزيـة تخدم كل التطبيقات بالمنظمة. 
حيث نوظف نظم المعلومات التقليدية لمعالجة المعاملات وإعداد التقارير اللازمة لاتخاذ القرارات، ولكن هذا ليس كافياً في بيئة الأعمال الجديدة

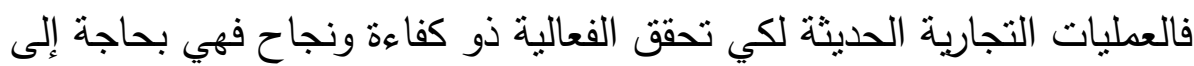

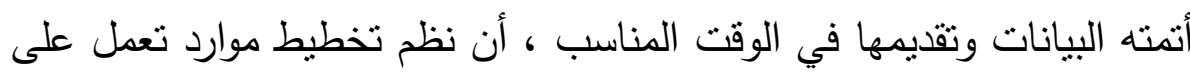

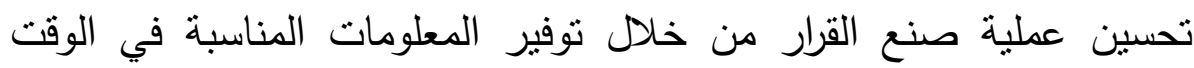
المناسب، علاوة على ذلك تحسن نظم تخطيط موارد من وظائف التخطيط والرقابة في العمليات التجارية. وبالإضافة إلى استخدام القطاعات المختلفة هذه النظم شجع نجاحها المنظمات

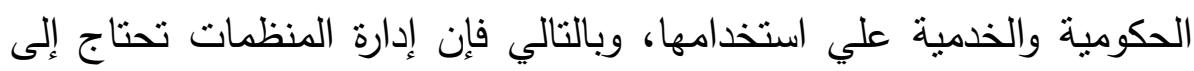

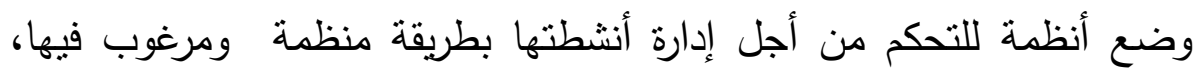

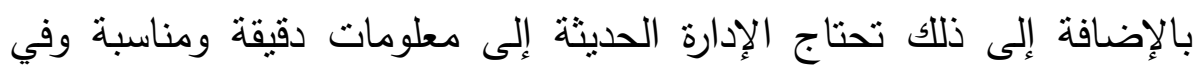

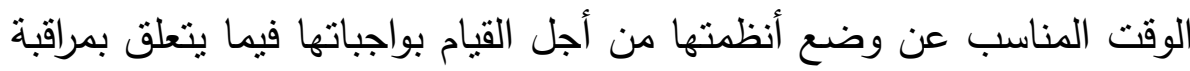
وتحسين نظم الرقابة الداخلية، وهذا بدوره يوفر لها اتخاذ القرار الصحيح

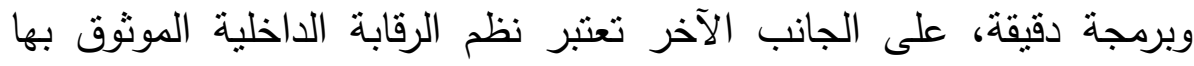

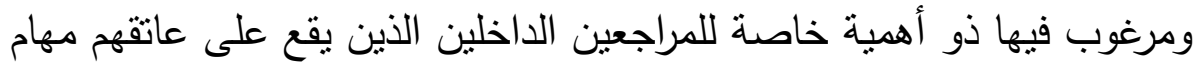

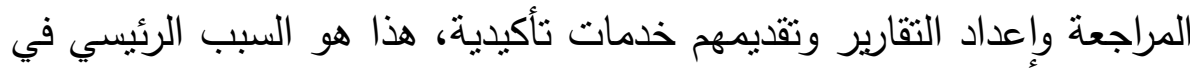

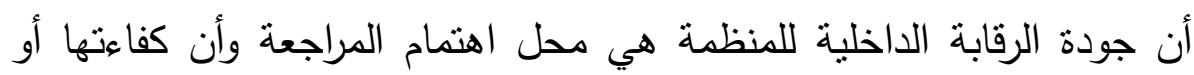

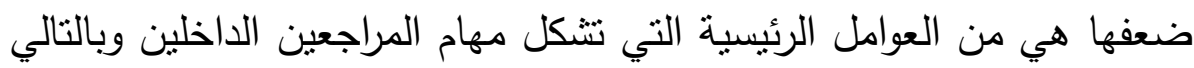

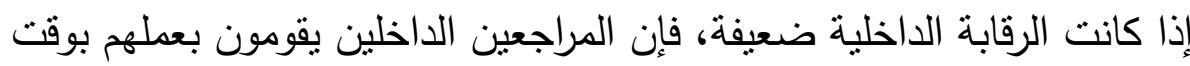
وتكلفة أكبر وسوف يواجه الدديرون صعوبات أكبر في صنع قرارهم.

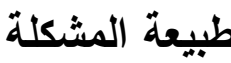

علي الرغم من أن تعريف المراجعة الداخلية قد تغير من عقد لآخر ، إلا أنها تواجه في الوقت الحاضر بعض الصعوبات المتعلقة بوظيفة المراجعة الداخلية

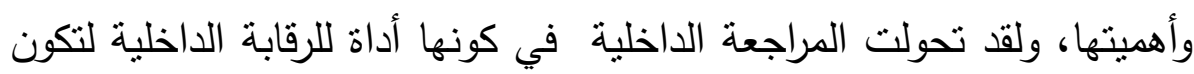


لفعالية الرقابة الداخلية، والتحقق من التلاعب أو مساعدة المراجع الخارجي

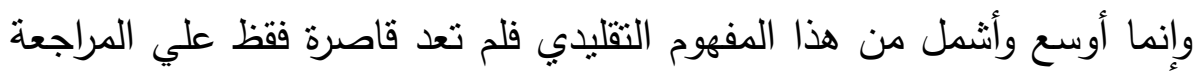
المنتظة أمتد دورها ليشمل أيضا التعريف بالمخاطر التي تتعرض لها الها المنظمة وتقديم الاستثارات اللازمة للإدارة العليا وحتى يمكن الاستفادة من كافة مزايا وظيفة المراجعة الداخلية يجب علي كافة الأطراف بالتتظيم الإدارة المحاسبين، ولجنة المراجعة أن يكون لديهم الفهم الكافي لأهمية الدور الذي تقوم به وظيفة

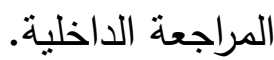

Barings ) نتيجة لتعرض العديد من المنظمات العالمية المعروفة منل

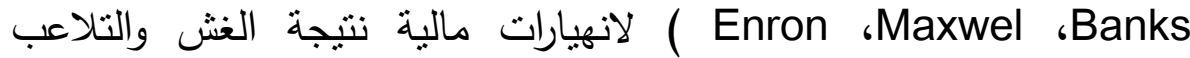
والأخطاء المحاسبية المتعدة في القوائم المالية والفساد الإداري والأخلاقي لإدارات المنظمات المختلفة فقد انعكس ذلك في تحقيق خسائر فادحة مما أدى الي عدم تقه أصحاب المصالح في هده النظم الدحاسبية والمالية والإدارية ويرجع هذا السبب الي ضعف النظام الرقابي المعمول به في تلاك المنظمات. وقد اثشارت احدى الدراسات التي تتاولت العلاقة بين المراجعة الداخلية وادارة المخاطر إلي عدم وجود التزام بممارسات المراجعة الداخلية يرجع ذلك إلي عدم لإنال الالتزام بالتعلم التنظيمي للمراجع الداخلي ومدي نتوافر ميثاق عل للمراجع الداخلي ومدي توافر الدعم المالي والإداري والمعنوي وتسهيلات المطلوبة لإدارة المراجعة الداخلية ثم استقلال إدارات المراجعة الداخلية. واثشارت احدي الدراسات التي تتاولت العلاقة بين المراجعة الداخلية ونظم

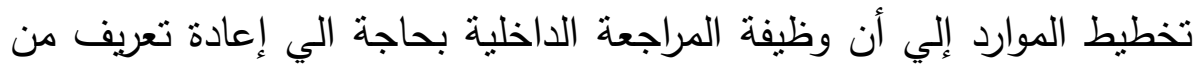
حيت النطاق و التركيز في ظل الإدارة الاستراتيجية من اجل التعامل مع مهام

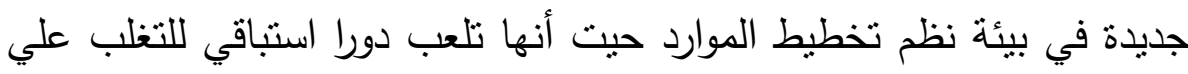
المشاكل المحتملة والناجمة عن الفصل بين الواجبات من خلال نقاسم خبراتها في مجال الرقابة الداخلية مع الاستثاريين وتعزيز فاعلية الرقابة الداخلية 
لمواجهة المشاكل الاحتيال والمعالجات غير المصرح بها.

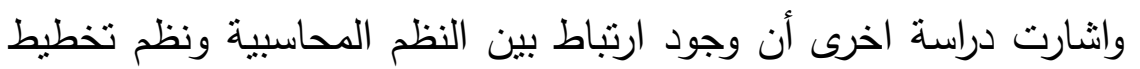

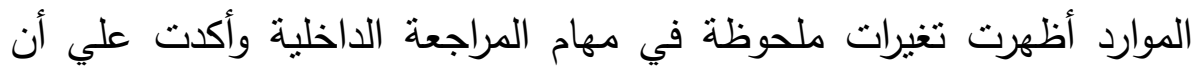
اعتماد نظم تخطيط الموارد يؤدي إلى زيادة في الرقابة الداخلية ويحسن من

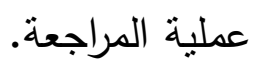
ومن خلال الدراسات السابقة يري الباحث أنهما اتفتا علي قصور دور

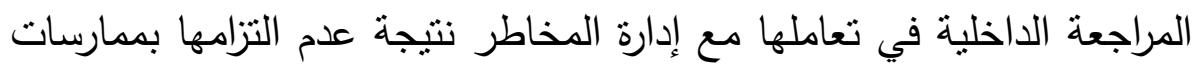
المهنة، وأن المراجعة الداخلية بحاجة الي إعادة تعريف من حيث النطاق عملها لإلها في ظل الإدارة الاستراتيجية لكي تواكب ولتتعامل مع نظم التكامل نظم تخطيط إنها موارد، حيث ركزت الدراسات السابقة علي العلاقة النبادلية بين المراجعة الداخلية وإدارة المخاطر، والعلاقة بين المراجعة الداخلية ونظم تخطيط الموارد

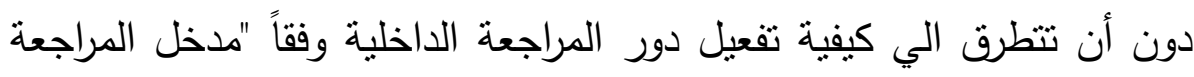
الداخلية علي أساس الخطر" في ظل بيئة نظم تخطيط الموارد لذا لذينسأل الباحث: هل ينعكس تطبيق نظم تخطيط الموارد علي تفعيل دور وظيفة

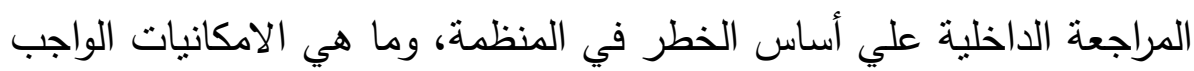

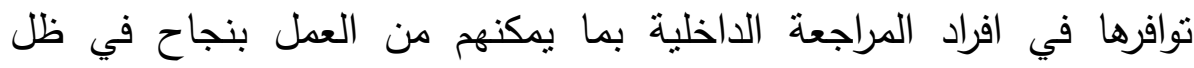

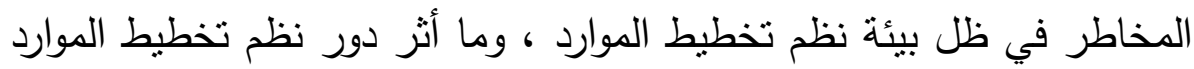
في دعم المراجعة الداخلية علي أساس الخطر.

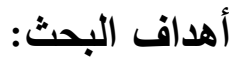

يهدف البحث إلي تفعيل وظيفة المراجعة الداخلية في ظل بيئة نظم تخطيط

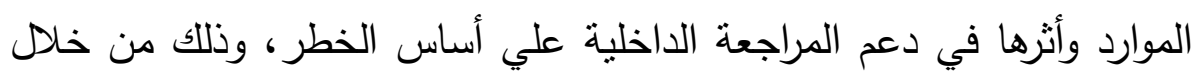
دراسة ميدانية علي المراجعين الداخليين وموظفي نظم المعلومات وموظفي إدارة المخاطر في القطاع المصرفي في ليبيا وبعض أعضاء هيئة التدريس في جامعات الليبية، ولتحقيق الهدف الرئيسي من البحث يقسم إلي الأهداف الفرعية 
التالية:

1- دراسة دور وظيفة المراجعة الداخلية في ظل بيئة نظم تخطيط الموارد من

حيث معرفة أهمية مساهتها في تطبيق نظم تخطيط الموارد، وطبيعة هذا

الدور ومدي فعاليتها، بالإضافة الي دراسة أثز التطبيق الفعلي لنظم

تخطيط الموارد كنظام معلومات يتسم بالجودة علي تحسين فعالية مدخل

المراجعة الداخلية علي اساس الخطر •

2- دراسة الامكانيات المطلوب توافرها في أفراد المراجعة الداخلية في بيئة نظم

تخطيط الموارد.

r- دراسة أثز تفعيل وظيفة المراجعة الداخلية في ظل بيئة نظم تخطيط الموارد

وأثرها في دعم مدخل المراجعة الداخلية علي اساس الخطر.

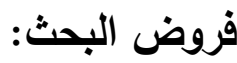

ا - لا يوجد اتفاق معنوي بين فئات البحث حول مدى تفعيل وظيفة المراجعة

الداخلية في ظل بيئة نظم تخطيط الموارد المنظمة.

r- لا يوجد اتفاق معنوي بين فئات البحث حول مدى ضرورة توافر مجموعة من المهارات العلمية والعملية لأفراد المراجعة الداخلية في بيئة نظم تخطيط

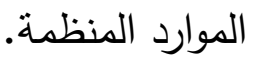

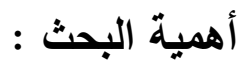

1- حيث يعتبر تطبيق تكنولوجيا نظم تخطيط الموارد كنظام معلومات متكامل

يتصف بالجودة و التطور السريع والمستمر في الموضوعات الحديثة وماله من تأثير علي العمليات المحاسبية نتيجة ما يحيط بها من مخاطر تتطلب تحديدها وتقدريها للمحافظة علي رقابة فعالة لتحقيق أمن وسلامة 
r- التطور في وظيفة المراجعة الداخلية كنشاط استشاري تأكيدي مضيف للقيمة ودورها الفعال في تحقيق الناجح عملية المراجعة الداخلية في ظل التل

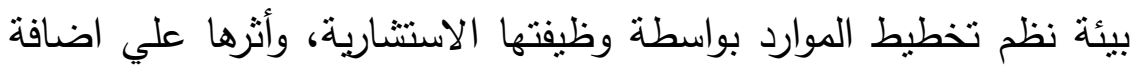

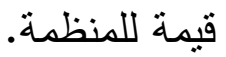

r- أن تفعيل وظيفة المراجعة الداخلية في ظل بيئة نظم تخطيط الموارد

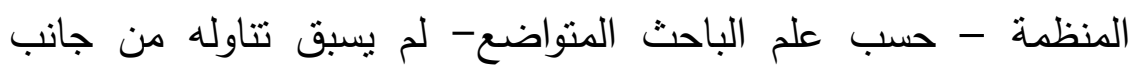
الباحثين وخاصتاً في البيئة الليبية ما دفع الباحث إلي محاولة تناوله.

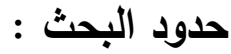

سوف يتم تطبيق البحث علي بعض المصارف العاملة في ليبيا، وذلك لاسنطاع الرأي المني والمتمثل في المراجعين الداخلين وموظفي نظم

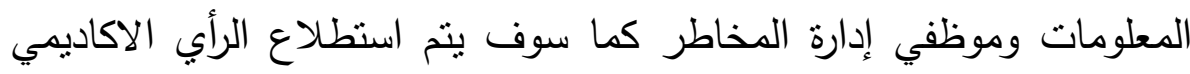
لبعض أعضاء هيئة التدريس في بعض الجامعات الليبية.

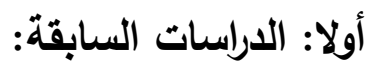
1- دراسة (Saharia.et.a,2008)

هدفت هذه الدراسة إلي التعرف علي أثز تطبيق نظم تخطيط موارد علي المراجعين الداخلين وذلك من الحد من الدخاطر التي تتعرض لها المنظمة. وقد توصلت الدراسة إلي أن المراجعين الداخلين يرون انخفاضا في المخاطر المالية والتشغيلية نتيجة استخدامهم لنظم تخطيط موارد(ERP) وزيادة في المخاطر التقنية نتيجة استخدامهم هذه نظم إلا أن هذه المخاطر يمكن الحد منها عن طريق تزويد الموظفين بالتدريب وتعليمهم علي هذه التقنية الحديثة. 


\section{r}

هدفت هذه الدراسة إلي بيان التطورات الحديثة في مهام المراجعة الداخلية المتعلقة بتقديم خدمات التأكيد والاستشارة وادارة المخاطر، وأثز ذلك علي استقلالية وموضوعية المراجع الداخلي، وذلك من خلا مراجعة الأدبيات الحديثة في المراجعة الداخلية ، واستعراض الأدبيات التي تتاولت تطور وظيفة

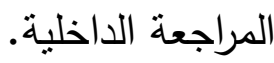

واوضحت الدراسة أن الخدمات التي تقدمها المراجعة الداخلية تستمد قيمتها من توافر الاستقلالية والموضوعية لاي أفراد المراجعة الداخلية لإضافة قيمة للمنظمة.

وتوصلت الدراسة إلى أهمية إعطاء المراجعة الداخلية الوضع المناسب في المنظمة لتمارس وظيفتها باستقلالية والتصرف بشكل موضوعي لتقييم ورصد إند القرارات وتصرفات الإدارة، لتقديم الاستشارات عن كفاءة وفاعلية الرقابة الداخلية وتقييم المخاطر، ويمكن للمراجع الداخلي مواجهة تهديدات الإدارة عن طريق لجنة المراجعة في المنظمة.

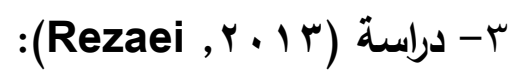

هدفت هذه الدراسة إلي تأثير تطبيق نظم تخطيط موارد علي جودة عملية المراجعة الداخلية وذللك باستخدام الأدلة التجريبية التي تم جمعها من المراجعين الداخلين من ذوي الخبرة في المنظمات التي تطبق نظم تخطيط موارد. و قد توصلت الدراسة إلي أن السيطرة على المخاطر تزداد بعد تطبيق نظم تخطيط موارد وهذا يعني علي المراجعين الداخلين تغير عملية المراجعة التقليدية إلي عملية المراجعة بتطبيق علي نظم تخطيط موارد . 


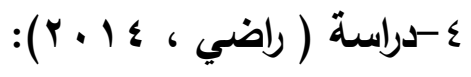

هدفت هذه الدراسة لوضع إطار مقترح لتفعيل دور المراجعة علي اساس

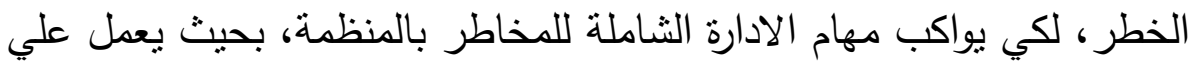

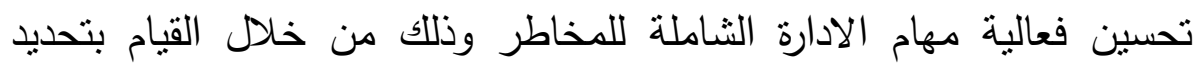

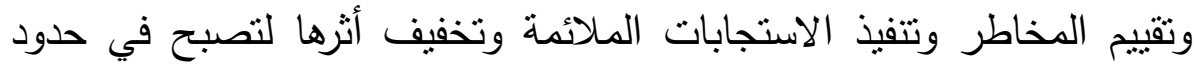

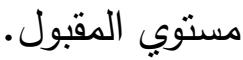

ولقد قام هذا البحث علي دراسة استقصائية وذلك باستخدام الاسلوب الإحصائي spss علي مجموعة من المدراء التتفيذين في المنظمات في البيئة المصرية ومدراء إدارات المراجعة الداخلية والمراجعين الداخلين العاملين فيهاء

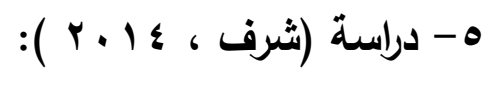

هدفت هذه الدراسة إلي تحليل العلاقة بين المراجعة الداخلية و نظم تخطيط

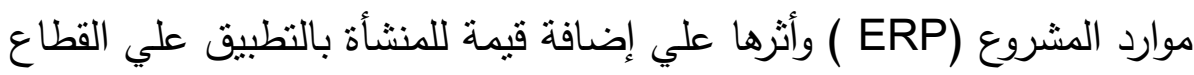

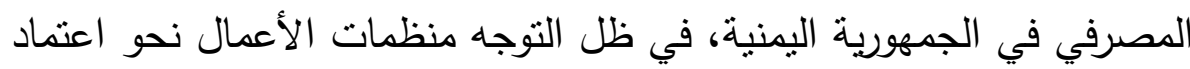

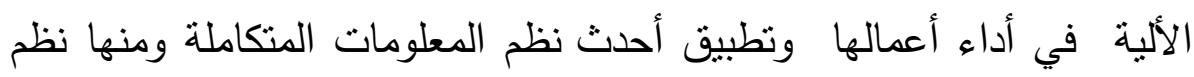
تخطيط الموارد لإحداث التكامل بين أنشطنها ووظائفها الداخلية والخارجية. وتوصلت الدراسة إلي أن المراجعة الداخلية نساهم في تقديم الاستثارات

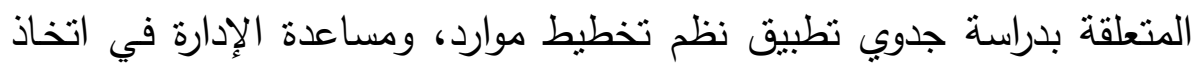

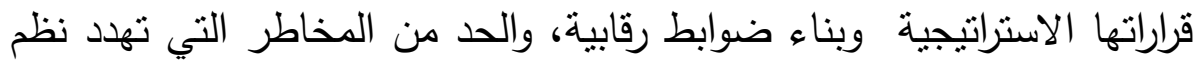
تخطيط الموارد نحو تحقيق هدف المنظمة، كما أن تطبيق نظم تخطيط موارد

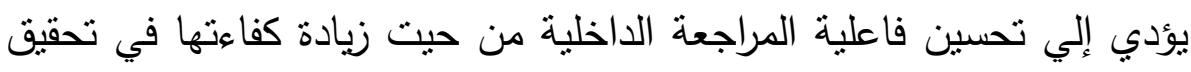
أهدافها بأقل جها وتكلفة وزيادة جودة تقاريرها. 
ثُانيا: محددات وركائز نظم تخطيط الموارد المنظمة: 1 - تعريف نظم تخطيط موارد المنظمة: * بأنه مجموعة برامج جاهزة تهدف إلي تكوين قاعدة بيانات واحدة لتوفير

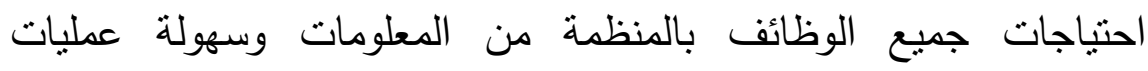
الاتصال وتدفق المعلومات بين الوظائف المختلفة.

r - خصائص نظم تخطيط الموارد المنظمة (ERP): ويمكن تلخيص أهم تلك الخصائص في الاتي:

* نظام متكامل : حيت تتصل بكل وحدات النظام بطريقة تكاملية معا في أن واحد في كل الإدارات المنظمة.

* قاعدة بيانات مشتركة : تمتز نظم تخطيط موارد (ERP ) بأن كافة تطبيقاتها تعمل معاً علي قاعدة مشتركة.

"الاتساق و التناسق: حيت نظهر نطبيقات نظم تخطيط موارد (ERP) بمظهر

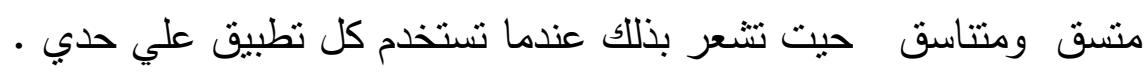
* اضافة قيمة للمنظمة وتخفيض التكاليف: من حيث تعزيز كفاءة إدارة العمليات الهامة للمنظمة مثل: تخطيط الإنتاج والمشتريات وإدارة المخزون والموردين وتسهيل تبادل البيانات المنظمة بعملائها عبر سلسلة التوريد بالثنكل الذي يؤدي إلي تخفيض تكاليف الاتتاج، إذ أن زيادة الطاقة الإنتاجية وزيادة

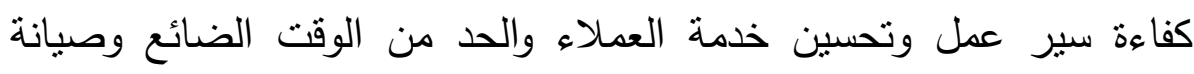

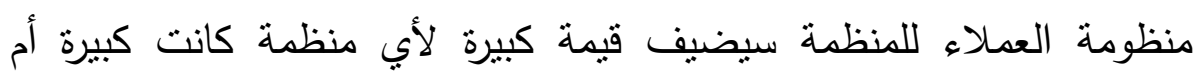
صغيرة. * تعزيز عملية صنع القرار الاستراتيجي وتخطيط الجيد للقدرات والموارد. 


\section{r- أهداف نظم تخطيط الموارد المنظمة:}

* تحقيق التكامل بين جميع الوظائف خلال كافة مراحل تثغيل العمليات. * تسهيل تدفق المعلومات والاتصال بين مختلف الوحدات الوظيفية وبالتالي إثباع احتياجات العاملين والعملاء.

* دعم عملية اتخاذ القرارات وإدارة أفضل لجميع الجوانب المالية. * تسهيل عمليات التجارة الإلكترونية وتحقيق المرونة والسرعة في استجابة وتكيف المنظمة مع أي متغيرات أو مؤثرات بالبيئة الخارجية.

*مواكبة التقدم التكنولوجي السريع ومواجهة المنافسين الذين قاموا بتطبيق نظم تخطيط الموارد المنظمة.

\section{؛ - مقومات نجاح تطبيق نظم تخطيط موارد المنظمة:}

*ملائمة النظام المقترح للعوامل البيئية المحيطة بالمنظمة، متل تحديد ومعرفة

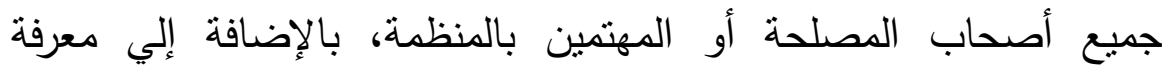
تقاليدهم وثقافتهم واحتياجاتهم قبل بدء تتفيذ النظم .

* توافر الثقة في المحيط الداخلي للمنظمة بالإضافة إلي توافرها بين المنظمة والثركة المصنعة للبرمجيات، وذلك بهدف التغلب علي مقاومة العاملين

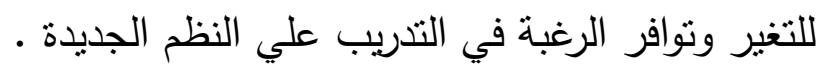

*"القيام بدراسة الجدوى الاقتصادية لنظم تخطيط الموارد قبل تتفيذها، وذللك بهدف قياس كل من التكاليف والهنافع الملموسة وغير الملموسة المتوقعة وتقييم صافي العائد المنوقع للحصول عليه نتيجة تطبيق هذه النظم. 
" وجود إدارة مؤهلة وكفء قادرة علي إدارة و تتفيذ مشروع تطبيق نظم تخطيط

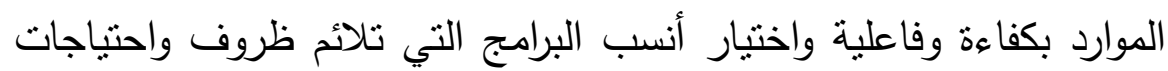
المنظمة، بالإضافة إلي استخدام أساليب إدارة المشروعات.

* دراسة مدي إمكانية تكامل نظم تخطيط موارد المنظمة مع النظم المستخدمة ومزايا تحقيق هذا تكامل والتأكد من وجود نرابط متبادل بين النظامين بهدف تجنب حدوث أخطاء في البيانات أو إعاقة تتفيذ العلميات.

ثالثا: المراجعة الداخلية علي أساس الخطر : 1- مفهوم المراجعة الداخلية علي أساس الخطر: *عرفها البعض" بأنها المنهجية نستخدم لتقديم تأكيد للإدارة المخاطر لتصبح في حدود المستوي الذي تقبله المنظمة.

"عرفت أيضا "بأنها طريقة مراجعة داخلية تساعد المراجع الداخلي علي أن

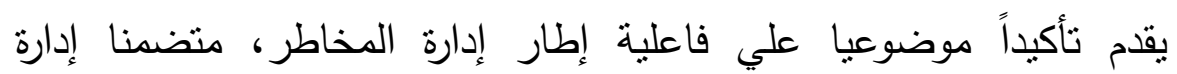
المخاطر الرئيسية.

r- الهلف من المراجعة الداخلية علي أساس الخطر: * إعطاء تأكيد علي أن العمليات التي تشتخدمها الإدارة لتحديد كل المخاطر . * اعطاء تأكيد علي أن المخاطر يتم تقيمها بدقة. * تقييم عمليات إدارة المخاطر للتأكد من أن الاستجابات التي استخدامها الإدارة لمعالجة المخاطر ملاءمة ومتوافقة مع سياسة المنظمة. * تقييم عملية إعداد التقارير عن المخاطر الأساسية من الدديرين للإدارة. 
*عرض عمليات إدارة المخاطر الأساسية للتأكد من أنها قد وضعت وتعمل

$$
\text { جيدا ويتم متابعتها. }
$$

\section{r- فوائد المراجعة الداخلية علي أساس الخطر :}

حيث تعتبر المراجعة الداخلية علي أساس الخطر من أكثر طرق التأكيد

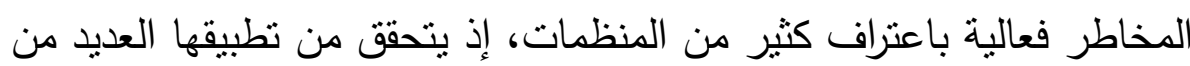

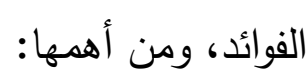

* توجيه موارد المراجعة الداخلية النادرة نحو تقييم الإدارة للمخاطر الرئيسية. * إطلاق تحذير واضح في حالة عدم فعالية إطار إدارة مخاطر المنظمة. * لفت الانتباه إلي المخاطر المبالغ في أنشطتها الرقابية، والمستهكة لموارد

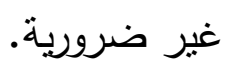
* مساعدة المنظمة علي تحديد حجم الموارد اللازمة لتقديم التأكيد الذي يطلبه

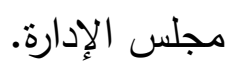

* تقيم تأكيد علي جميع أجزاء عملية إدارة الدخاطر . * تدعيم مسئولية الإدارة نحو إدارة المخاطر في كل مرحلة، ومساعدة المنظمة في زيادة قوتها وقدرتها علي مواجهة المخاطر الرئيسية والتعامل معها.

رابعا: طبيعة العلاقة بين المراجعة الداخلية في ظل بيئة نظم تخطيط الموارد وأثرها في دعم مدخل المراجعة الاخلية علي أساس الخطر :

1 - دور نظم تخطيط الموارد في دعم المراجعة الداخلية علي أساس الخطر: تعتبر نظم تخطيط الموارد (ERP) نظاما متكاملا ومترابطا يعتمد علي الحاسب الالي ويقوم بمعالجة جميع العمليات المنظمة من خلال مجموعة من لفابع 
النطبيقات الفرعية للإنتاج المخازن والمشتريات، البيع والنسويق، إدارة شئون الأفراد، الشئون المالية والمحاسبية، ونظم المعلومات، وهي برامج مهيأة للقيام بأنثطة مختلفة للمنظمات تعمل في مجالات مختلفة الصناعية والتجارية والزراعية وغيرها، فهي برامج تعمل علي ربط جميع الإدارات والوظائف ويمدها بالاحتياجات الخاصة بها من المعلومات، وبالتبعية تمكن المنظمة من التخطيط السليم لاستخدام مواردها أفضل استخدام ممكن.

حيث تساعد نظم تخطيط الموارد علي تحقيق الانسيابية للعمليات وتمكين المنظمة من الاستجابة سريعا للتغيرات في السوق، وهذا ما اكدت عليه دراسة(Shang and Seddon,2002) بتصنيف المنافع الناتجة من استخدام

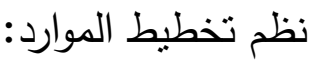
* المنافع التشغيلية. المنافع الاستراتيجية. المنافع الإدارية. * المنافع التنظيمية. المنافع المرتبطة بالبنية التحنية لنظام المعلومات. حيث يؤدي تطبيق نظم تخطيط الموارد إلي تحسين وتسهيل عمليات التحليل الإضافي للمعلومات المحاسبية وذلك من خلال التطبيقات المنقدمة والملحقة

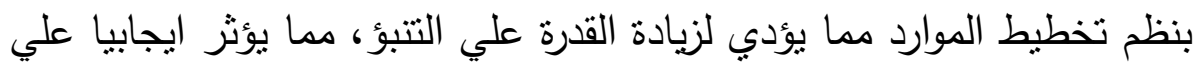

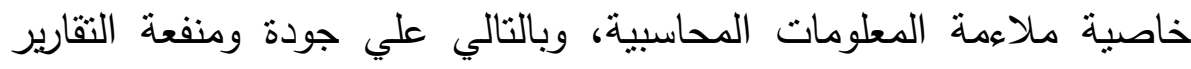
المالية وغير المالية، وبالتبعية يؤثر ايجابيا علي عملية أنخاد القرار والاستغلال

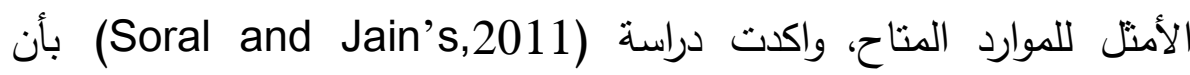
التكنولوجيا المعلومات المتمثلة في نظم تخطيط الموارد استخدام الجديدة يرفع من جودة المراجعة الداخلية ونظام الرقابة الداخلية في المنظمات.

r - اثر تطبيق نظم تخطيط الموارد علي تحسين كفاءة المراجع الداخلي: 
* المساعدة في تحقيق أهداف المنظمة من خلال توفير احتياجات المستويات الإدارية من المعلومات اللازمة لمباشرة وظائف التخطيط والرقابة والتقييم

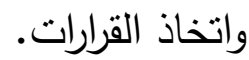

* توصيل البيانات والمعلومات إلي المستويات الإدارية بسهولة ووضوح وبأقل تكلفة ممكنة وفي الوقت المناسب وبالثكل الذي يساعد علي تقييم الأداء. * توفير احتياجات الجهات الخارجية من المعلومات. * تحقيق التكامل والترابط والتتاسق بين الأنظمة الفرعية المختلفة من جهة والأنظمة الكلية والبيئة الخارجية من جهة تانية ولنية. خامساً: إجراءات الدراسة الميدانية: استكمالاً لما عرضت له الدراسة من رؤى نظرية حول الإطار الدفهوم

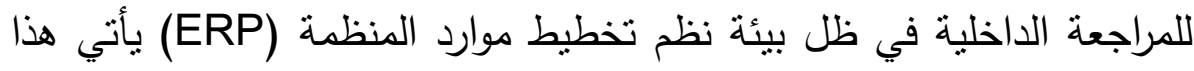
الجزء للتعرف على أثر تطبيق نظم تخطيط الموارد ERP كنظام للمعلومات

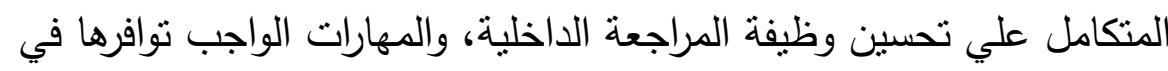
أفراد المراجعة الداخلية في ظل بيئة نظم تخطيط الموارد ERP. حساب معاملات ارتباط درجة كل مفردة بالدرجة الكلية للححور الذب تتنمي إليه، وجاءت النتائج كما هي مبينة بجدول (1) وذللك على النحو الآتي: 


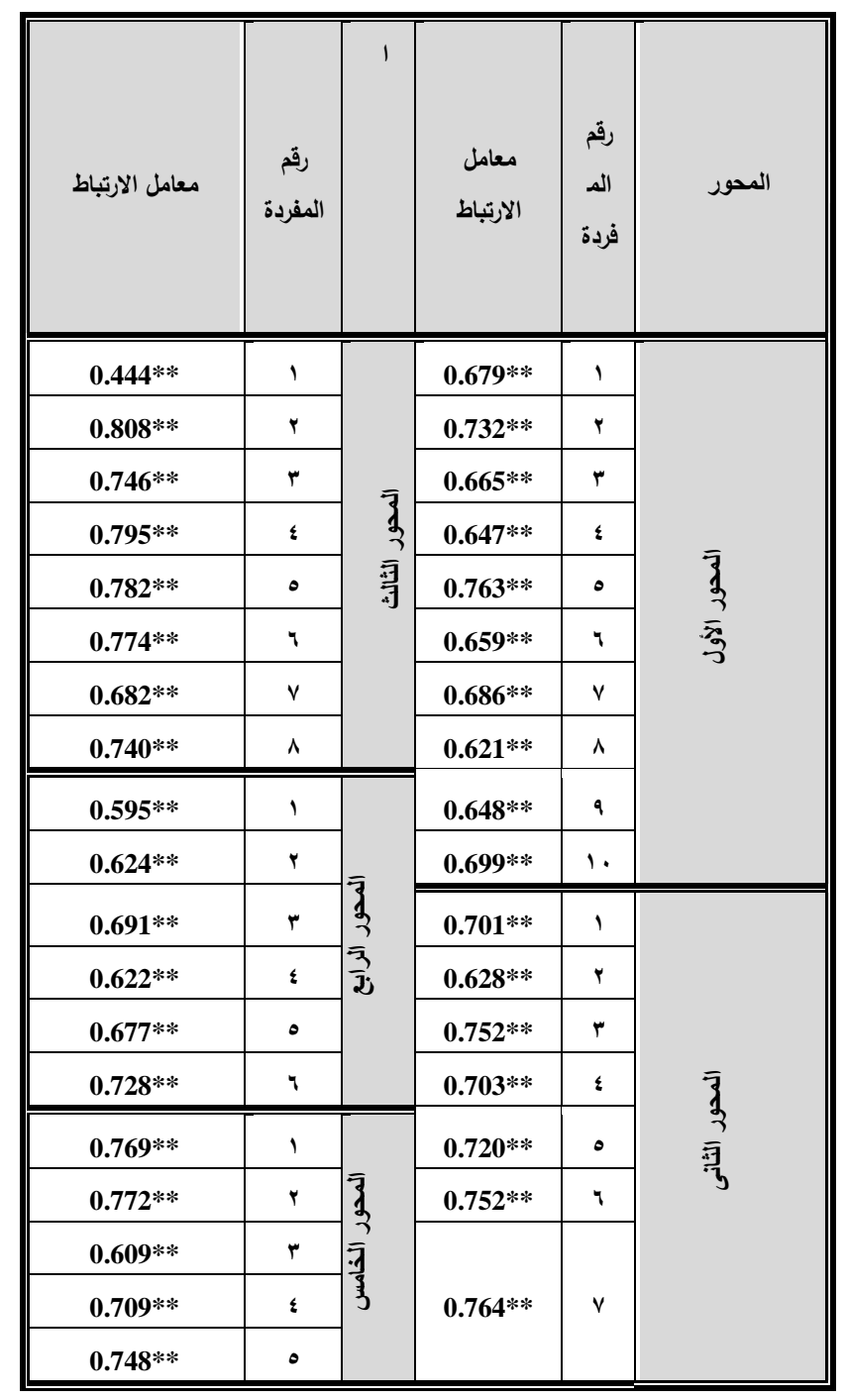

$$
\text { تعنى أن الارتباط دال عند مستوى (1 ...). }
$$

يتضح من جدول(1) أن جميع قيم معاملات الارتباط كانت موجبة ودالة يتضح من جدول(1) أن جميع قيم معاملات الارتباط كانت موجبة ودالة عند مستوى (1 (. . )؛ حيث تراوحت قيم معاملات ارتباط درجة كل مفردة بالدرجة الكلية للمحاور كالآتي: 
• بالنسبة للمحور الأول تراوحت قيم دعاملات الارتباط لمفرداته بين( آT. · )

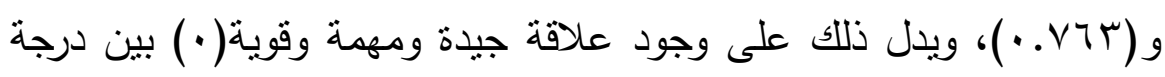
كل مفردة والدرجة الكلية لهذا الدحور .

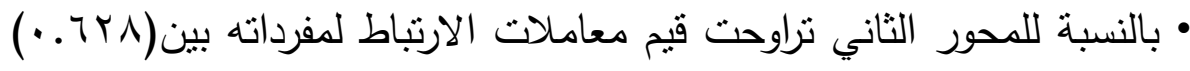

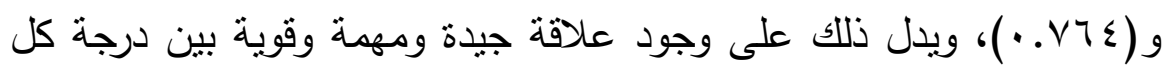

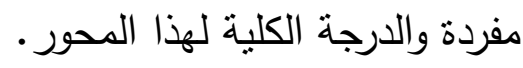

• بالنسبة للدحور الثالث تراوحت قيم دعاملات الارتباط لمفرداته بين(؟ ؟ ـ. ·)

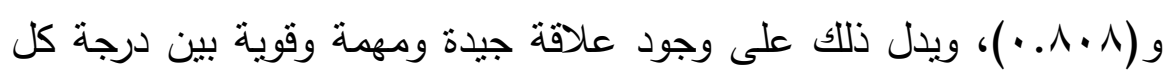

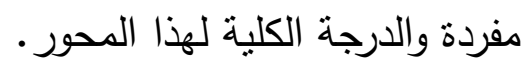

• بالنسبة للمحور الرابع تزاوحت قيم معاملات الارتباط لمفرداته بين(090. •)

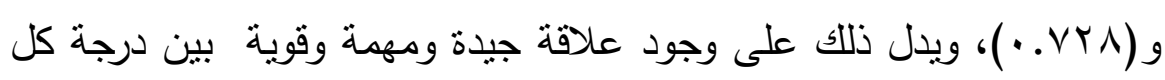
مفردة والدرجة الكلية لهذا المحور .

•بالنسبة للمحور الخامس تراوحت قيم معاملات الارتباط لمفرداته بين(9 ـ 7. • )

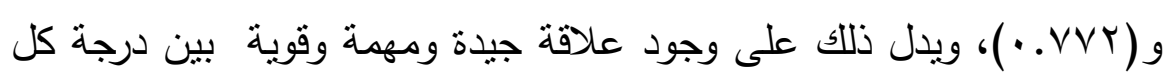
مفردة والدرجة الكلية لهذا المحور .

** تم حساب معاملات ارتباط درجة كل محور بالدرجة الكلية للاستبانة كما هو مبين في جدول رقم (r) الاتي: 


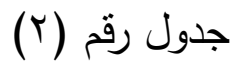

\begin{tabular}{|c|c|c|}
\hline معامل ألفام & عدد العبارات & المحور \\
\hline 0.870 & 10 & المحور الأول \\
\hline 0.843 & 7 & المحور الثاني \\
\hline 0.870 & 8 & المحور الثالث \\
\hline 0.736 & 6 & المحور الرابع \\
\hline 0.771 & 5 & المحور الخامس \\
\hline 0.946 & 36 & الاستبانة كاملة \\
\hline
\end{tabular}

يتضح من جدول(r) أن جميع قيم معاملات الارتباط موجبة ودالة عند مسنتى (1 (.))، حيث تراوحت قيم معاملات ارتباط درجة محاور الاستبانة

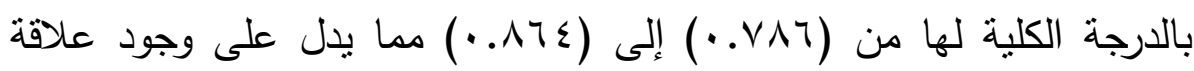
قوية بين درجة كل محور والدرجة الكلية للاستبانة. قام الباحث بحساب ثبات الاستبانة بطريقة ألفا كرو نباخ، وكانت النتائج كما

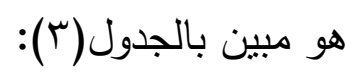

\begin{tabular}{|c|c|c|}
\hline معامل ألفا & عدد العبارات & المحور \\
\hline 0.870 & 10 & المحور الأول \\
\hline 0.843 & 7 & المحور الثاني \\
\hline 0.870 & 8 & المحور الثالث \\
\hline 0.736 & 6 & المحور الرابع \\
\hline 0.771 & 5 & المحور الخامس \\
\hline 0.946 & 36 & الاستبانة كاملة \\
\hline
\end{tabular}


يتضح من نتائج جدول أن قيم الثبات لمحاور الاستبانة تراوحت بين (ד VT...، عالية .AV. عالية ومقبولة إحصائيا.

\section{سادسا: النتائج والتوصيات:}

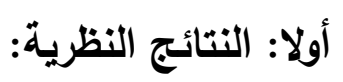

ERP يؤدي استخدام نظم التكامل المتمنلة في نظم تخطيط موارد المنظمة -

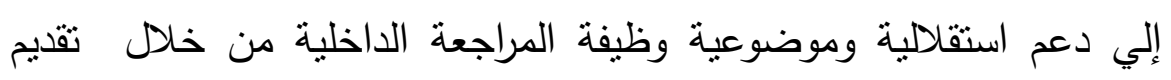

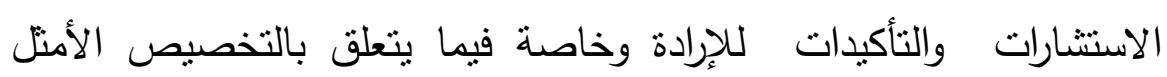
للموارد المتاحة، وذلك بالتركيز علي وجود قاعدة بيانات مركزية مشتركة ومتكاملة تتنج معلومات تتصف بأنها فورية ييني عليها المراجع الداخلي رأيه

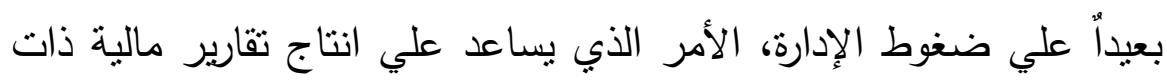
جودة ومنفعة عالية.

- يتوقف مدى أهمية المراجع الداخلي في تطبيق نظم تخطيط الموارد علي

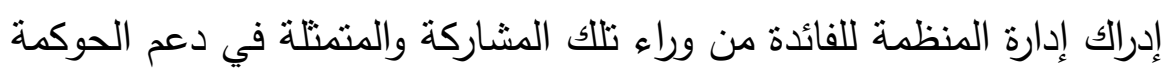

$$
\text { الجيدة وتحقيق منطلبات الإدارة الاستراتيجية. }
$$

- ضرورة دعم الإدارة العليا لتطبيق هذه النظم، ونشر ثقافة التغيير لدى العاملين في المنظمة.

- يعد من أهم عوامل نجاح نظم تخطيط موارد المنظمة إيجاد حلقة تواصل

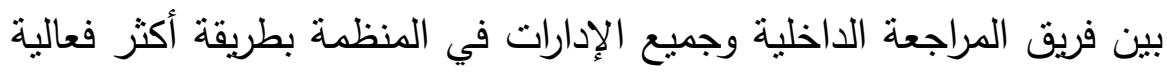
من ناحية، وبائعي البرامج الجاهزة من ناحية. 
- العمل علي تعزيز المهارات التقنية والخبرة العملية في مجال تكنولوجيا

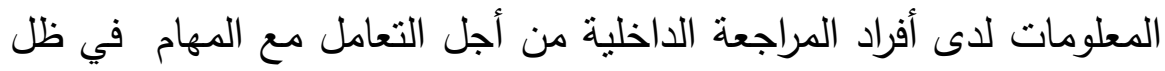

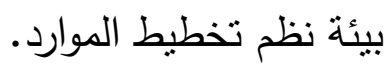

- استخدام مدخل المراجعة الداخلية علي اساس الخطر بالثكل الكامل لتقديم معلومات فورية لجميع الأطراف المهتمة كأحد منطلبات تطبيق هذه النظم.

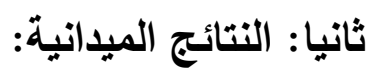

- قبول الفرض الاول ، يتضح من اختبارات الفرض الاول وجود اتفاق معنوي بين فئات البحث حول مدى تفعيل وظيفة المراجعة الداخلية في ظل بل بيئة نظم تخطيط الموارد المنظمة.

- قبول الفرض الثاني، يتضح من اختيارات الفرض الثاني وجود اتفاق معنوي بين فئات البحث حول مدى ضرورة نوافر مجموعة من المهارات العلمية

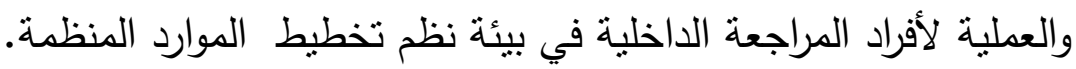

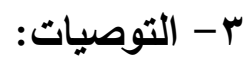

- ضرورة الاتجاه نحو تطبيق منهجية المراجعة المستمرة ( مدخل المراجعة

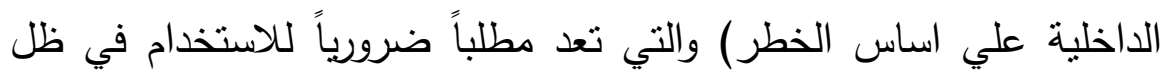
تطبيق نظم تخطيط المواردERP، حيث تمكن المنظمة من تخفيف المخاطر التي تصاحب تطور تكنولوجيا المعلومات، وأن تتوفر الرغبة لاى المنظمات

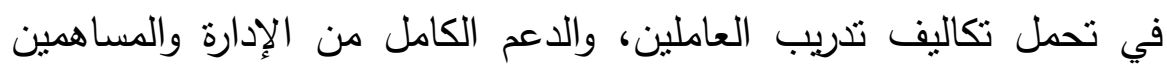
ل المنهجية المراجعة المستمرة.

- إنشاء معهد للمراجعين الداخليين الليبيين، يتمكن من إصدار المعايير المنظمة للعمل في البيئة الاعمال الليبية 
المراجع

اولا: المر اجع العربية:

- ابر اهيم جمال الدين محمود،" إطار مقترح لتحقيق متطلبات نظام تخطيط موارد المنظمة(ERP) بالتطبيق علي جامعة المنصورة، رسالة ماجستير كلية

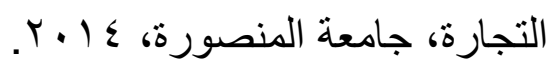

ـ احمد جمال الدين ، مدونة نظم: كيف تصبح استشاري لنظم تخطيط الموارد

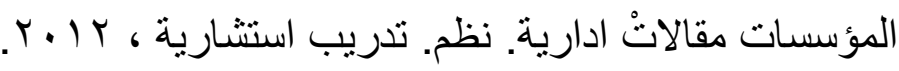

- شحاتة السيد شحاتة " دراسات متقدمة في الرقابة والمر اجعة الداخلية وفقاً

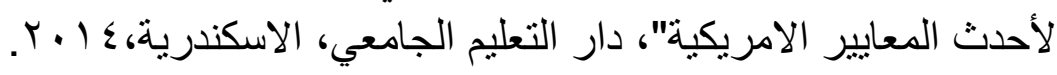

- عبده احمد عبده عشتي ، "اطار مقترح لرفع مستوي أداء المر اجعة الداخلية

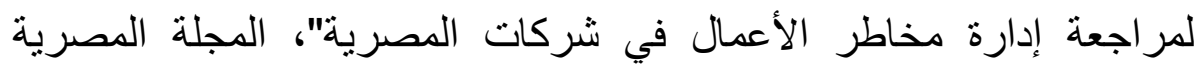

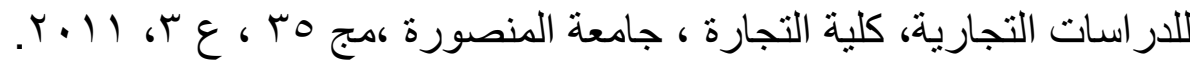

- كارم شرف شرف عثمان، دراسة تحليلية للعلاقة بين المراجعة الداخلية

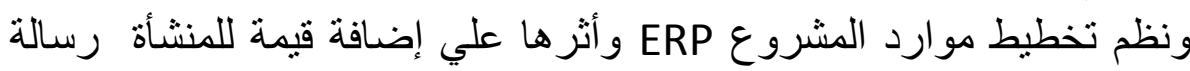

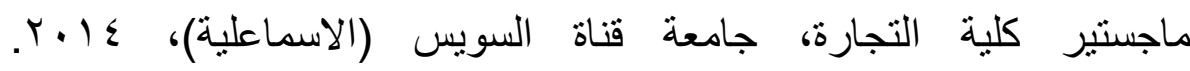

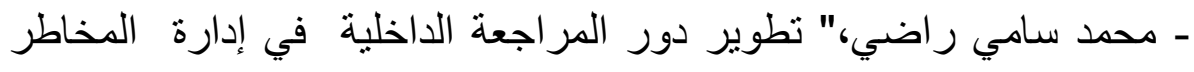

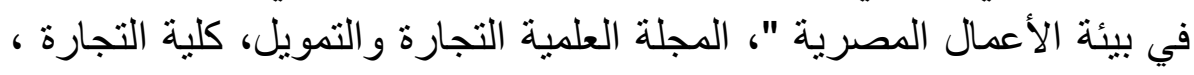

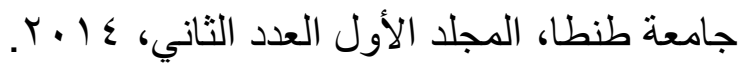

- محمد مصطفي جمعة، " أثر تطبيق نظم تخطيط موارد المشروع (ERP) علي نظم الرقابة المحاسبية وفعالية مخرجات النظام المحاسبي - دراسة

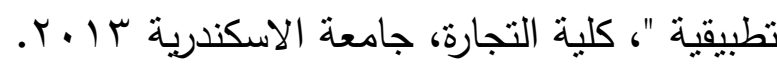

ERP محمد محمد محمد ابراهيم، " اثز تطبيق نظم تخطبط موارد المشروع علي ممارسات المحاسبة الإدارية في منشآت الأعمال في جمهورية مصر 


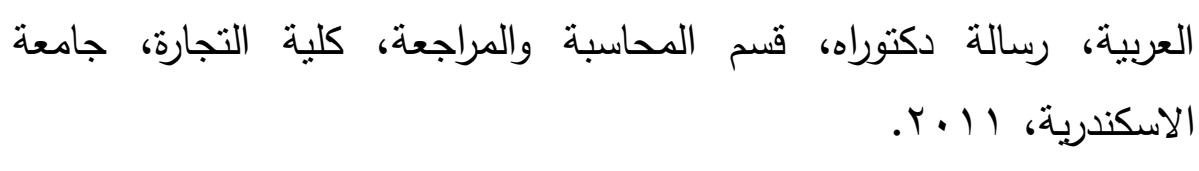

\section{ثانيا: المراجع الأجنبية :}

- Aditya Saharia. Robert Tucker \& Bruce Koch " ERP SYSTEMS AND INTERNAL AUDIT" Issues in Information Systems . VOL IX, No. 2, 2008.PP: 578-586 .

- cain.J.An approach to implementing risk based internal auditing, the institute of internal auditors UK and Ireland march, 2006 , p:4.

- Debreceny, R.S," Embedded Audit Module in Enterprise Resource Planning System : Functionality, Journal of Information Systems", Vol.19, No.2,2011,p:10.

- Dominic,S,B, Nonna, M,B, "The internal audit function Perceptions of internal audit roles effectiveness : and evaluation", Management Auditing Journal ,Vol,26,No1,7, 2011,p:606.

- Galani, Despina, Efthymios Gravas and Antonios Stavropoulos " ERP Benefits and Firm Performance in

Greece Galani", Gravas, Stavropoulos 2010.PP:143-152.

- Griffiths, "Risk Based Internal Auditing" 2006.p:1.Hashem Valipour and Javad Moradi," The Impact of Enterprise Resource Planning (ERP) on the Internal Controls Case Study: Esfahan Steel Company ", European Journal of Social Sciences ISSN 1450-2267 Vol.28.No,2, 2012,p:231.

- Haider. H .Madani, " The role of internal auditing in ERP based organizations", Journal of Accounting \&.Organizational Change .Vol .5.No.4.2009.pp:514.

- Nader rezaei " Enterprise Resource Planning ERP Software Implementation Impacts on the Auditing Activities 
"Journalof Applied Business and Finance Researches .Vol 2, No 3.2013.pp: 90 - 96.

- Soral.G and Jain.M, " Impact of ERP System on Auditing and Internal control ", The International Journals Research Journal of Social Sciences and Management, Vol. 1, No.4,2011,pp:16-23.

-Shang,S, and P,B,Seddon, "Assessing and managing the Benefits of Enterprise System: the Business Managers Perspective ", Information System Journal ,12,4,2002,p:280.

- Spathis \& Kanellou, " Auditing in enterprise system environment a synthesis", Journal of Enterprise Information Management. Vol .24.No.6.2011.pp: 495-519.

-"Spathis and Constantinides, S, " Enterprise Resource Planning Systems Impact on Accounting Processes, Business Process Management Journal, Vol .10.Iss.2, 2004, $\mathrm{p}: 246$.

- Schniederjans ,M.J. and Kim,G.C. " Implementing ERP System with Total Quality Control and Business Process :Reengineering: Survey Results", International Journal of Operations \& Production management, Vol .23, ISS 4, January,2003,PP:418-429.

- Stewart, Subramanian, "Internal audit independence and objectivity emerging research opportunities" Managerial Auditing Journal Vol.25.No.4.2010, pp : 328 -360.

- Turner ,L,D, and V, Owhoso" Use ERP Internal Control Exception Reports to Monitor and Improve Controls ", Management Accounting Quarterly(Spring)10,3,2009,P48. 